\title{
High concentration measurement of mixed particle suspensions using simple multi-angle light scattering system
}

\author{
S. Buaprathoom ${ }^{a^{*}}$, S. Pedley ${ }^{b}$ A. D. Prins ${ }^{a}$ and S. J. Sweeney ${ }^{a}$ \\ ${ }^{a}$ Advanced Technology Institute, Faculty of Engineering and Physical Science, University of Surrey, \\ Surrey GU2 7XH, UK \\ ${ }^{\mathrm{b}}$ Postgraduate Medical School, Faculty of Health and Medical Sciences, University of Surrey, Surrey \\ GU2 7XH, UK
}

\begin{abstract}
A simple multiple-angle light scattering system was developed for the differential measurement of particle concentrations in suspension even in high concentration where multiple scattering effects are significant based on size. The system combines multiple-angle detection to collect scattered angle dependent light intensities, and Partial Least Square Regression method (PLS-R) to compose the predictive models for analyzing scattered signal obtain concentrations of samples under investigation. The system was designed to be simple, portable and inexpensive. It employs a diode lasers (red AlGaInP-based) as a light source and a silicon photodiode as a detector and optical components, all of which are readily available. The technique was validated using $1.1 \mu \mathrm{m}$ and $3.0 \mu \mathrm{m}$ polystyrene latex beads in both mono-dispersed and poly-dispersed suspensions. The measurement results showed good agreement between the measured results and reference values. Their deviations from the reference values are $2.4 \%$ and $1.5 \%$ relating to references' concentrations of $1.3 \times 10^{8}$ and $1.2 \times 10^{7}$ particles $/ \mathrm{ml}$ for $1.1 \mu \mathrm{m}$ and $3.0 \mu \mathrm{m}$ in mono-dispersed solutions and $2.3 \%$ and $3.5 \%$ relating to references' concentrations of $1.1 \times 10^{8}$ and $4.4 \times 10^{5}$ particles $/ \mathrm{ml}$ for $1.1 \mu \mathrm{m}$ and $3.0 \mu \mathrm{m}$ in mixed solutions, respectively. This system is a compact but high performance system allowing multiple particle sizes in high concentration to be measured simultaneously.
\end{abstract}

Keywords: particle concentration measurement, light scattering, high concentration, mixed particle concentration

\section{INTRODUCTION}

Particle concentration measurements for suspension are important processes in quality control in many systems and manufacturing lines such as pharmaceutical, water quality system etc. There are many methods used to measure the particle concentration in suspension, for instance using ultrasonic attenuation method ${ }^{1-2}$, ultrasonic impact vibration method $^{3}$, volumetric flask method ${ }^{4}$, and also light scattering techniques. As the latter technique are easy to perform and has many attractions; noninvasive nature, high sensitivity, potential for real-time detection and low maintenance, therefore the light scattering have been widely used to determine the properties of particle in suspension including concentration. The intensity of scattered light $(I)$ are dependent on the scattering angle $(\theta)$ incident wavelength $(\lambda)$ and size $(x)$, shape and the optical properties of the system (specifically, the index of refraction $(n)$ of the particle relative to the suspension medium. In fundamental light scattered by an individual spherical particle, the scattering pattern are provided by Mie theory ${ }^{5}$ which is a function as per equation 1.

$$
I_{\text {individual }}=f(\theta, \lambda, x, n)
$$

In practice, the light scattering pattern from suspension is determined by a collection of particles in the suspension medium. Therefore, scattered light intensity from the suspension is also a function of particle concentration $(c)$ in the suspension.

$$
I_{\text {suspension }}=f(\theta, \lambda, x, n, c)
$$

*s.buaprathoom@surrey.ac.uk; phone +44 (0)1483689404

Optical Sensing and Detection II, edited by Francis Berghmans, Anna Grazia Mignani, Piet De Moor, Proc. of SPIE Vol. 8439, 843923 - (c) 2012 SPIE · CCC code: 0277-786X/12/\$18 · doi: 10.1117/12.922287 
In low concentration (single scattering), the scattered light intensity can be considered from addition of scattered light intensity by each particle. Consequently, the scattered light intensity directly proportion to the particle concentration. For suspension in high concentration, multiple scattering effects are significant causing the scattered signals to vary in indirect proportion to the particle concentration ${ }^{6}$.

As the light scattering intensity is determined by the properties of the scattering particles as per equation 2 , light scattering techniques have been applied to obtain the scattering phase function to infer the particle's size ${ }^{7-9}$, refractive index ${ }^{10-11}$, and concentration ${ }^{12-13}$. However, there are some limitations of measurement for particle suspension at high concentration due to multiple scattering effects ${ }^{14-15}$. The common way to avoid this effect is to dilute the sample so that the sample transmittance is above $0.95^{16}$. The effect of multiple scattering to scattered light when light scattered from high concentration suspension is shown in Figure1.
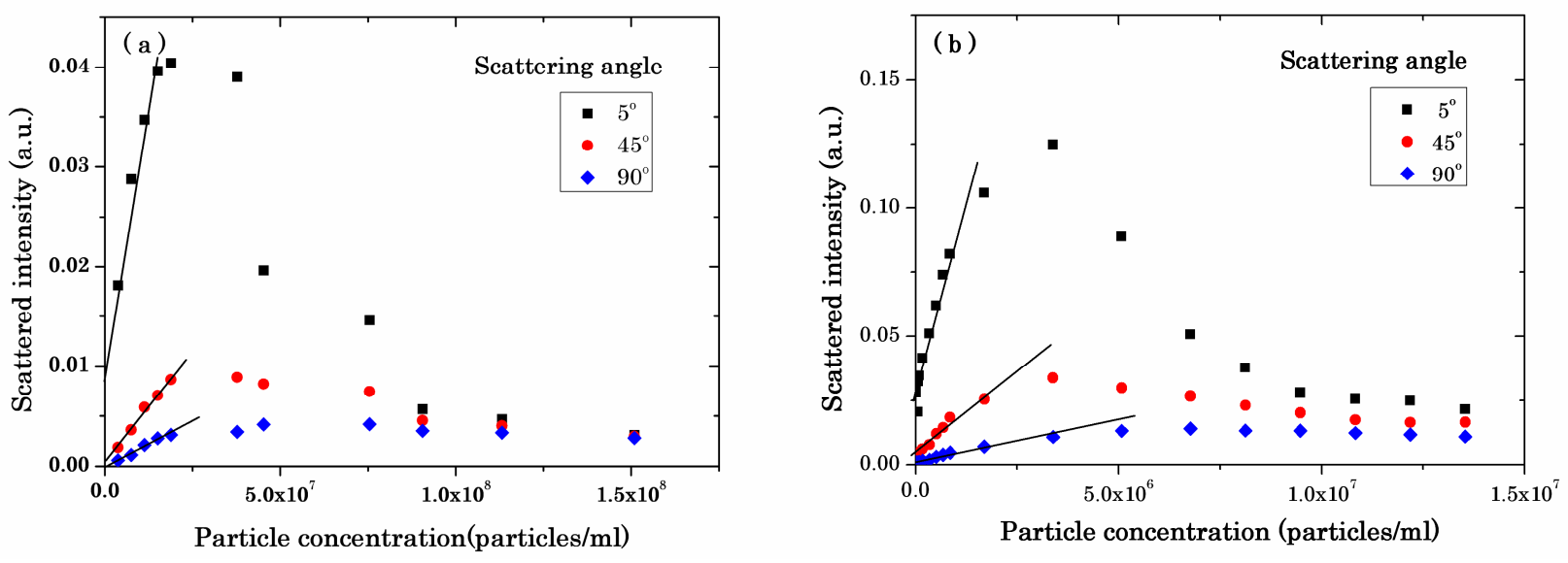

Figure 1 Scattered light intensities of mono-dispersed polystyrenes aqueous suspension at the different concentrations at $5^{\circ}, 45^{\circ}$, and $90^{\circ}$ scattering angles for (a) $1.1 \mu \mathrm{m}$. (b) $3.0 \mu \mathrm{m}$ diameter.

Figure 1 shows that the scattered intensities are not linearly dependent to entire ranges of the particle concentrations. In the low concentration, the scattered intensities increase monotonically with the concentration (solid straight line) which means scattered intensity increased with the concentration of scattering particles. However, for high concentrations the scattered intensities either decreased or were stable due to multiple scattering in the suspensions. Consequently, measurement of particle concentration by using a basic nephelometer, which typically detects the scattered light at a fixed scattering angle (normally measure at 90 degree because this angle is considered to be the least sensitive to particle size) and simple linear regression cannot apply to the system with high concentration where the multiple light scattering effects are significant. Moreover, in many industrial applications or in academic research, it is not only mono-dispersed suspensions needing to be measured but also poly-dispersed suspensions. Most of the light scattering techniques have been applied to mono-dispersed systems ${ }^{17-19}$. Others that have been applied to poly-dispersed systems determine only particle size distributions but not concentration directly ${ }^{20-21}$.

Here we describe the application of a multiple-angle light scattering system in small scattering angles for measuring the concentration of high concentrated and mixed particles in suspension. The system design combines multiple-angle detection, to collect scattered angle dependent light intensities, and multivariate data analysis techniques using a partial least square regression (PLS-R) method to generate a predictive model from calibration samples and predict the concentration of unknown samples. The system was designed to be simple, portable and inexpensive using a low power diode laser as a light source, Si photodiodes for detection and optical components, all of which are readily available. 


\section{EXPERIMENTAL METHOD}

\subsection{Experimental set up}

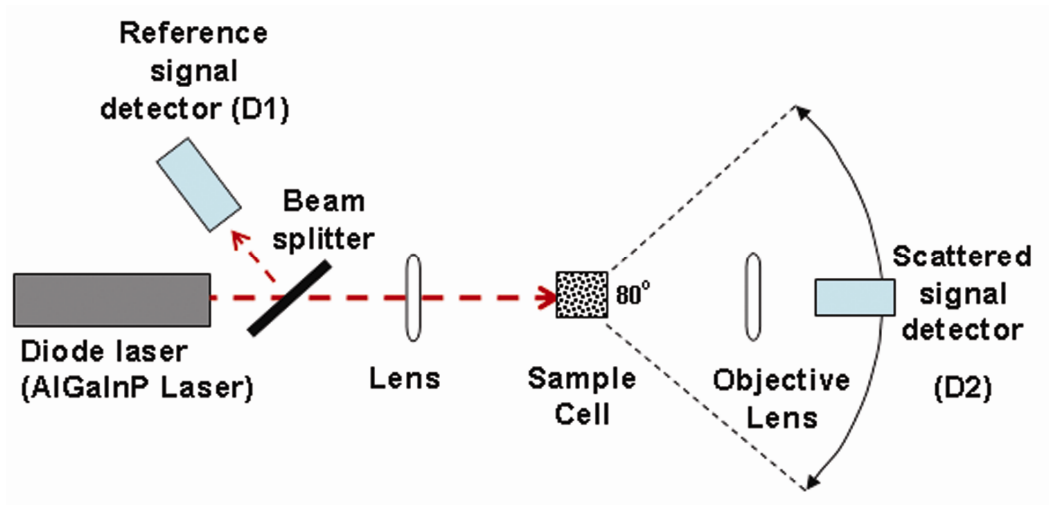

Figure 2 Schematic drawing of experimental set up.

The design of the multiple-angle light scattering system is shown in figure 2. A red diode laser (AlGaInP laser) was used as the light source. The power to the diode and the temperature of the diode were controlled using a source-meter (KEITHLEY 2420) and temperature controller (LDT-5910B), respectively. The diode was set to emit a $5 \mathrm{~mW}$ parallel polarised light at $658.4 \mathrm{~nm}$-wavelength (measured by an optical power meter (ANRITSU ML910B) and an optical spectrum analyzer (AQ 6315A)). A beam splitter (glass slide) was used to divide the light beam into a reference signal detected by a detector D1 and an incident signal. A thin lens $(\mathrm{f}=75 \mathrm{~mm})$ focused and directed the incident light beam onto the centre of a sample cell (glass cuvette with a cross-section of $10 \mathrm{~mm} \times 10 \mathrm{~mm}$ and a height of $50 \mathrm{~mm}$ ). An objective lens (20x, 0.40NA) focused the scattered light signal onto the signal detector (D2). Silicon photodiodes (AEPX65) with a buffer circuit were used as detectors both the scattered (D2) and reference signals (D1). The scattered signal detector was mounted on a rotating base which has angular resolution 0.2 degree and was able to be controlled via Labview ${ }^{\mathrm{TM}}$ software. The apparatus was protected from external electromagnetic interference (which affected the performance of the detectors), airborne particles, surrounding light, and air-flow (which caused temperature fluctuation of the laser) by covering with a metal box.

\subsection{Experimental samples}

The performance of the proposed set up was validated using mono-dispersed and poly-dispersed systems. The experimental samples of both mono-dispersed and poly-dispersed suspensions were polystyrene latex beads (Supplied by Sigma-Aldrich, UK) suspended in de-ionized water. 22 samples of polystyrene latex bead solutions of $1.1 \mu \mathrm{m}$ diameter (LB11) with concentration of $10^{6}-10^{9}$ particles/ml (Cal LB11 No.1-22) and 27 samples of polystyrene latex bead solutions of $3.0 \mu \mathrm{m}$ diameter (LB30) with concentration of $10^{4}-10^{7}$ particles/ml (Cal LB30-No.1-27) were prepared as calibration samples to generate a predictive model for mono-dispersed system. In case of poly-dispersed system, a predictive model was generated from 30 mixed solutions between LB11 with concentration of $10^{5}-10^{8}$ particles $/ \mathrm{ml}$ and LB30 with concentration of $10^{2}-10^{5}$ particles/ml (Cal Mixed LB No.1-30). Test samples were prepared with different concentration from the calibration samples for each particle size for mono-dispersed solutions and poly-dispersed solutions as shown in table 1 and table 2 , respectively. 
Table 1 Concentrations of the test samples for mono-dispersed system

\begin{tabular}{cc|cc}
\hline Sample Name & $\begin{array}{c}\text { LB11 particle } \\
\text { concentration } \\
\text { (particle } / \mathrm{ml})\end{array}$ & Sample Name & $\begin{array}{c}\text { LB30 particle } \\
\text { concentration } \\
\text { (particle } / \mathrm{ml})\end{array}$ \\
\hline LB11-No.1 & $1.28 \times 10^{8}$ & LB30-No.1 & $1.15 \times 10^{7}$ \\
LB11-No.2 & $8.30 \times 10^{7}$ & LB30-No.2 & $7.45 \times 10^{6}$ \\
LB11-No.3 & $3.77 \times 10^{7}$ & LB30-No.3 & $3.39 \times 10^{6}$ \\
LB11-No.4 & $1.28 \times 10^{7}$ & LB30-No.4 & $1.15 \times 10^{6}$ \\
LB11-No.5 & $5.23 \times 10^{6}$ & LB30-No.5 & $4.69 \times 10^{5}$ \\
\hline
\end{tabular}

Table 2 Concentrations of the test samples for poly-dispersed system

\begin{tabular}{ccc}
\hline Sample Name & $\begin{array}{c}\text { LB11 particle } \\
\text { concentration } \\
\text { (particle } / \mathrm{ml})\end{array}$ & $\begin{array}{c}\text { LB30 particle } \\
\text { concentration } \\
\text { (particle } / \mathrm{ml})\end{array}$ \\
\hline Mixed LB No.1 & $5.28 \times 10^{6}$ & $4.40 \times 10^{5}$ \\
Mixed LB No.2 & $1.13 \times 10^{7}$ & $1.69 \times 10^{5}$ \\
Mixed LB No.3 & $1.09 \times 10^{8}$ & $2.12 \times 10^{4}$ \\
Mixed LB No.4 & $3.66 \times 10^{7}$ & $2.12 \times 10^{4}$ \\
\hline
\end{tabular}

\subsection{Experimental operation}

The apparatus as shown in figure 2 was operated as follows. The light beam from the diode laser was divided into two parts by the beam splitter. The reflected light was detected by the photodetector (D1) and used as a reference feedback signal for stabilizing the power of the laser. The transmitted light was focused by the thin lens onto the centre of the sample cell containing the experimental sample prepared in the manner described above. Light entering the cell was scattered and absorbed by the particles in suspension. The scattered light was focused by the objective lens onto the photodetector (D2), which was rotating to collect the scattered signals. As the scattered signal is the most sensitive in forward scattering for the range of used particle sizes, and to avoid transmitted light, the scattered signals in this set up were designed to be collected through an angle from $5^{\circ}$ to $35^{\circ}$ in steps of one degree. The Labview ${ }^{\mathrm{TM}}$ program was used to control the operation of the apparatus and to collect both reference and scattered signals via multimeters.

To obtain the particle concentration of the test samples, calibration models or predictive models were composed from angle dependent scattered signals of the calibration samples of known concentration by using a multivariate data analysis method. The scattered signals from the unknown test samples were predicted based on these calibration models.

\subsection{Multivariate data analysis}

The multivariate data analysis technique used in this experiment was Partial Least Square Regression (PLS-R) method. The PLS-R is particularly useful when we need to predict a set of dependent variables or determination values $\left(Y_{1}, Y_{2}, Y_{3}, \ldots\right)$ from a set of independent variables or measurement values $\left(X_{1}, X_{2}, X_{3}, \ldots\right)$. It is used when typically the measurement of $\mathrm{Y}$ may be expensive, difficult, dangerous, labour intensive, etc. The basic if this technique is to construct a multivariate regression model or predictive model using the information from standard data or calibration data to find the $\mathrm{Y}$-relevant pattern in $\mathrm{X}$ by successively extracting latent factors from both $\mathrm{X}$ and $\mathrm{Y}$ such that correlation between the extracted factors is maximized. The model is then subsequently used on new $\mathrm{X}$ measurements to predict new Y-values ${ }^{22}$. The schematic of the concept of the multivariate data analysis is shown in figure 3. 


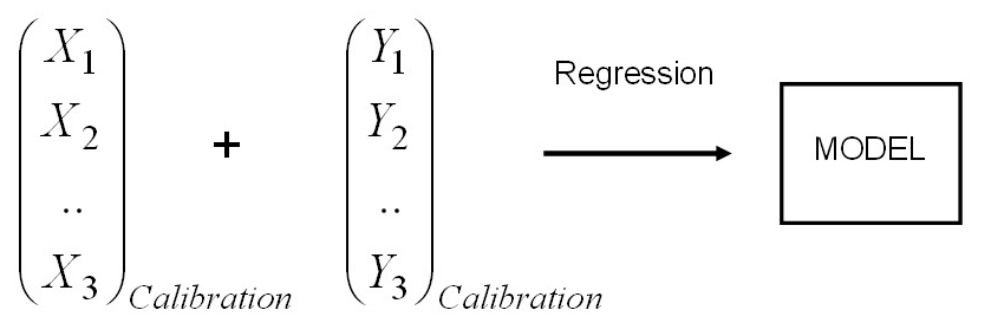

(a)

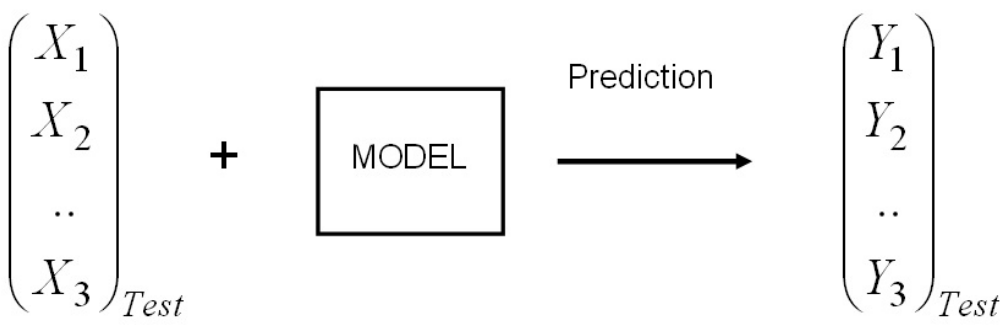

Figure 3 (a) Establishing a multivariate regression model from known $\mathrm{X}$ and $\mathrm{Y}$ data. (b) Using the multivariate regression model to predict new Y values

For the validation of the proposed system the incident wavelength and refractive index of samples were controlled, therefore in order to construct the predictive models the angle dependent scattered intensities were used as the sets of independent variables or measurement values for both the mono and poly-dispersed systems and the concentrations of LB11 and LB30 in mono-dispersed suspension were constituted as dependent variables or determination values in the mono-dispersed system whilst the concentrations LB11 and LB30 in mixed solutions were set as dependent variables in the poly-dispersed system. The angle dependent scattered signals from the five test samples for each size of polystyrene latex beads in mono-dispersed solutions and four test samples for poly-dispersed solution were measured and used as independent variable for predicting the particle concentration of each size of the test samples.

\section{RESULTS AND DISCUSSIONS}

\subsection{Mono-dispersions}

The calibration samples of both LB11 and LB30 in mono-dispersed suspensions were measured by the proposed scattering system. Their angle dependent scattered intensities are shown in figure 4a for LB11 and figure $4 \mathrm{~b}$ for LB30.
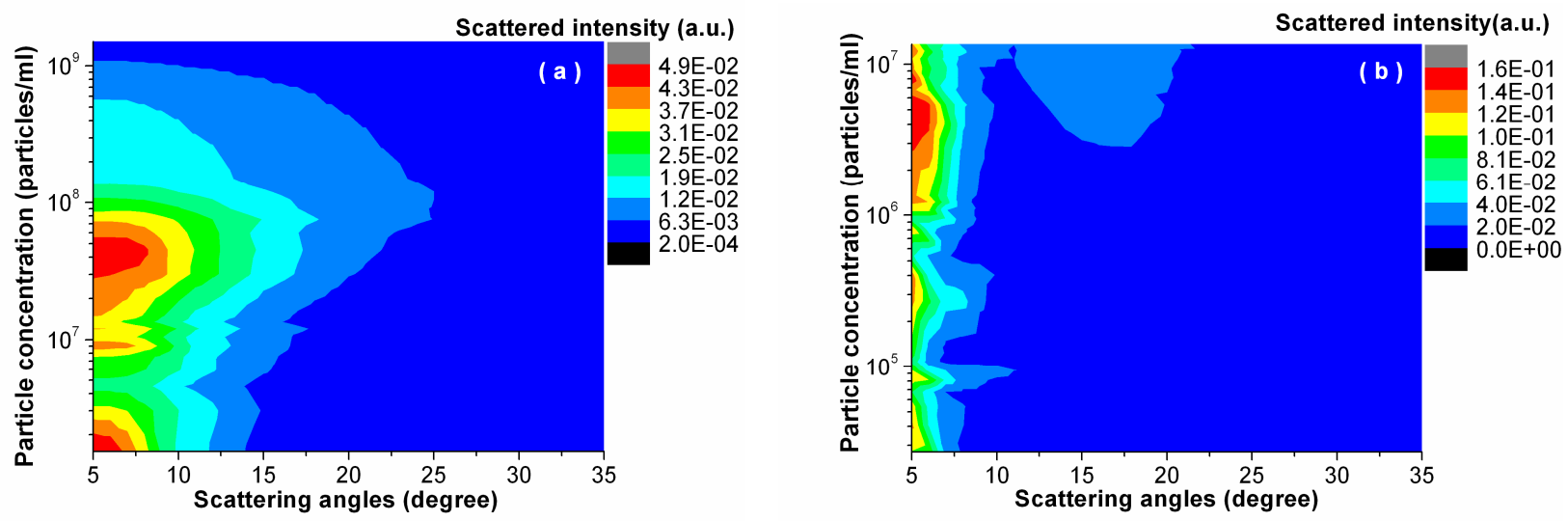

Figure 4 The graphs of angle dependent scattered intensities of calibration samples for (a) LB11 and (b) LB30 in monodispersed suspensions 
The measured scattered signals of the calibration samples were composed to the predictive models using the PLS-R method. The results of the regression of the predictive models are presented in figure $5 \mathrm{a}$ for LB11 and $5 \mathrm{~b}$ for LB30.
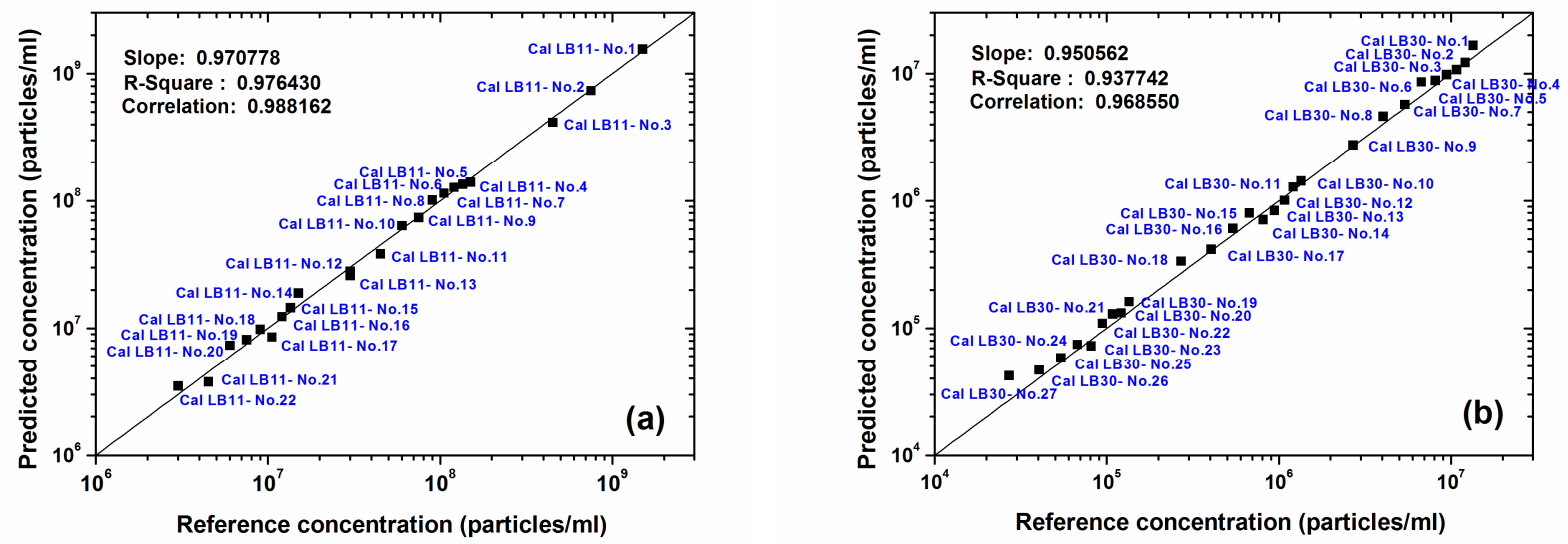

Figure 5 The results of the regression of the predictive models for (a) LB11 and (b) LB30 in mono-dispersed suspensions

From figure 5, the regression of the predictive models in monodispersed samples found that the correlation between reference concentrations and predicted concentration of the calibration samples were 0.988 and 0.969 for the models of LB11 and LB30 mono-dispersed suspensions, respectively. These correlation values inferred that there were very good relations between the extracted factor from dependent and independent variables that meant the constructed models could reasonably have been used to predict new observations.

The test samples scattered signals were analyzed to predict their concentrations based on the predictive models. The scattered intensities of the test samples and the concentration prediction results are shown as figure 6a and 6b for LB11 suspensions and figure $7 \mathrm{a}$ and $7 \mathrm{~b}$ for LB30 suspensions.
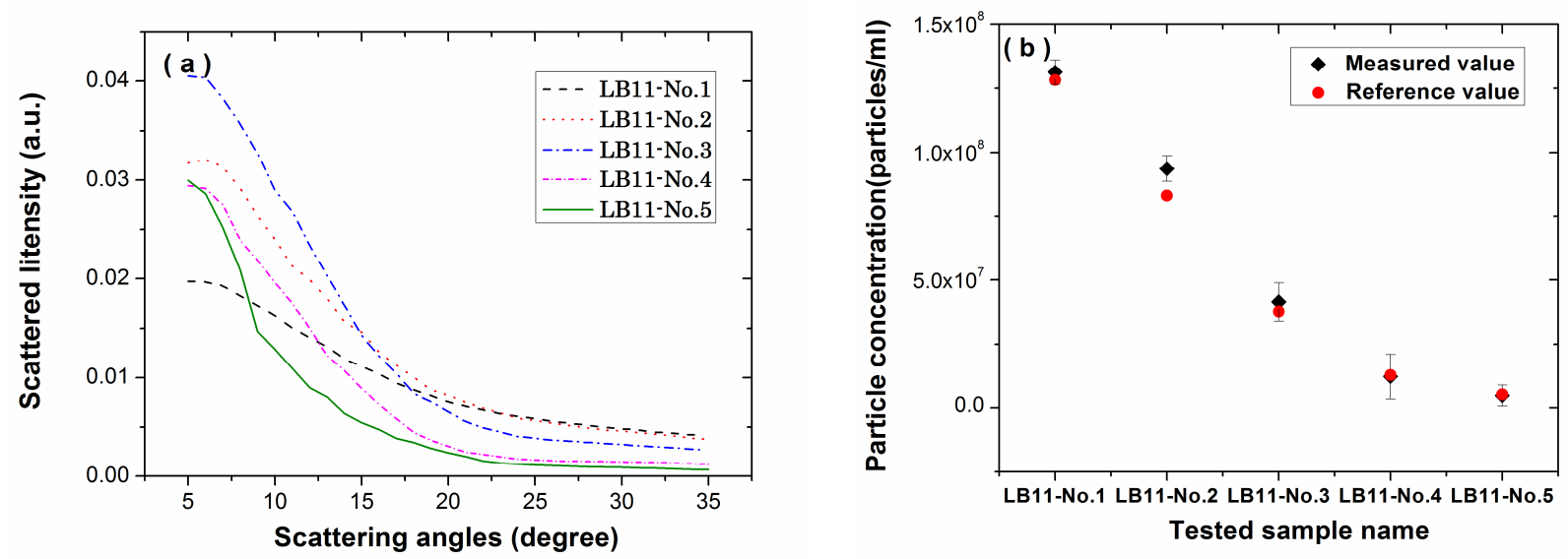

Figure 6 (a) Scattered intensities from test samples of $1.1 \mu \mathrm{m}$ polystyrene latex beads in mono-dispersed suspensions (LB11). (b) Predicted results for test samples of $1.1 \mu \mathrm{m}$ polystyrene latex beads in mono-dispersed solutions. 

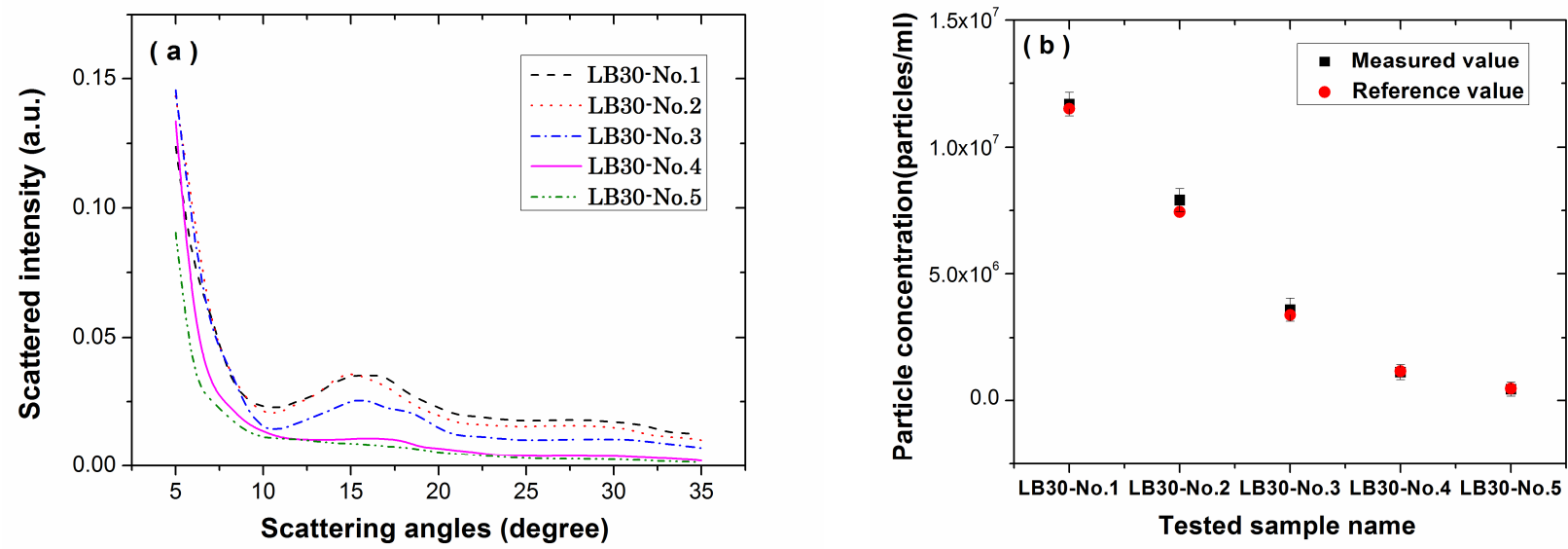

Figure 7 (a) Scattered intensities from test samples of $3.0 \mu \mathrm{m}$ polystyrene latex beads in mono-dispersed suspensions (LB30). (b) Prediction results for test sample of $3.0 \mu \mathrm{m}$ polystyrene latex beads in mono-dispersed suspensions.

The prediction results of test samples LB1 1 as figure $6 \mathrm{~b}$ and the LB30 as figure $7 \mathrm{~b}$ showed good agreement between the predicted results and reference values. The predicted results (particles $/ \mathrm{ml}$ ) and their standard deviation (in bracket) were $1.31 \times 10^{8} \quad\left(4.78 \times 10^{6}\right), \quad 9.35 \times 10^{7}\left(4.99 \times 10^{6}\right), \quad 4.14 \times 10^{7}\left(7.46 \times 10^{6}\right), \quad 1.23 \times 10^{7}\left(8.76 \times 10^{6}\right) \quad$ and $4.85 \times 10^{6}\left(4.10 \times 10^{5}\right)$ for LB11-No.1 to LB11-No.5 and $1.17 \times 10^{7}\left(4.63 \times 10^{5}\right), 7.90 \times 10^{6}\left(4.45 \times 10^{5}\right), 3.60 \times 10^{6}$ $\left(4.49 \times 10^{5}\right), 1.11 \times 10^{6}\left(2.94 \times 10^{5}\right)$ and $4.46 \times 10^{5}\left(2.72 \times 10^{5}\right)$ for LB30-No1 to LB30-No5, respectively. The lowest error of the results from the control reference values were $2.4 \%$ and $1.5 \%$ relating to references' concentrations of $1.3 \times 10^{8}$ and $1.2 \times 10^{7}$ particles $/ \mathrm{ml}$ for $1.1 \mu \mathrm{m}$ and $3.0 \mu \mathrm{m}$ size. The results demonstrated that the proposed system can be used to measure the concentration of the mono-dispersed suspensions even in high concentration condition where the multiple scattering effects are significant.

\subsection{Poly-dispersions}

For the poly-dispersed system, the mixtures of LB11 and LB30 suspensions were prepared as the calibration and test samples described in section 2.2. The angle dependent scattered signals of the calibration samples were collected for generating a predictive model by the proposed set up. The relative graphs of the scattered intensities, scattering angles and the concentrations of the calibration samples are shown in figure 8 .
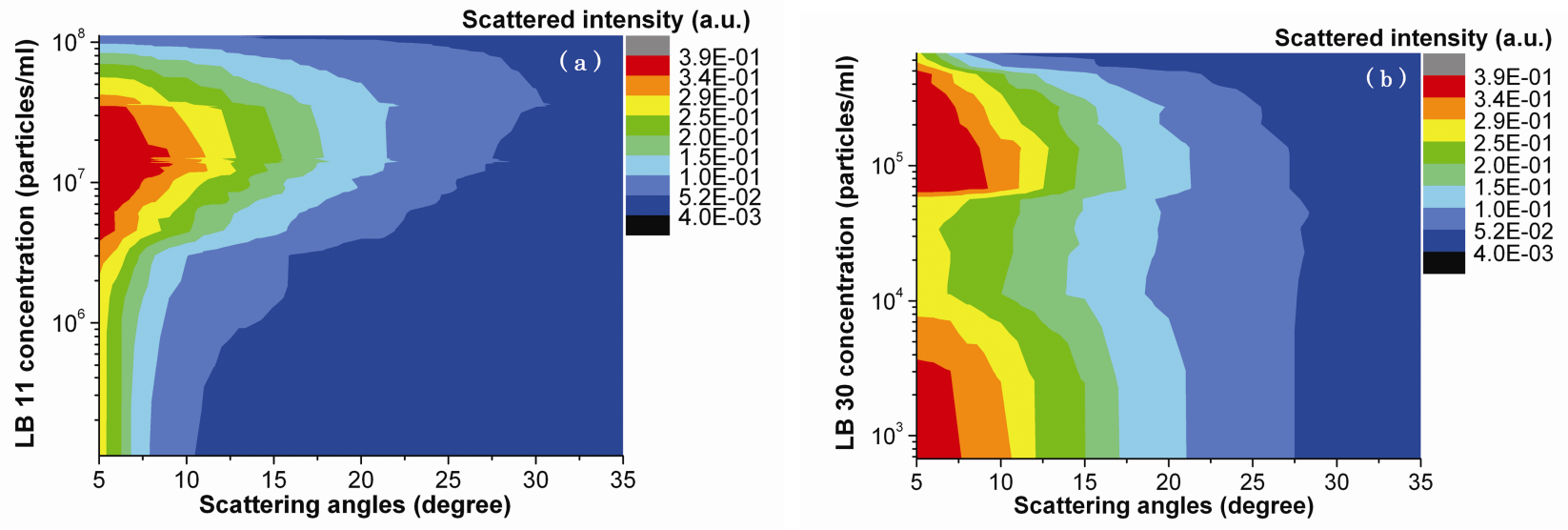

Figure 8 The graphs of angle dependent scattered intensities of calibration samples for (a) LB11 and (b) LB30 in polydispersed suspensions 
The predictive model for the poly-dispersed system was also composed using the PLS-R method. For constructing the predictive model in the poly-dispersed system there were two dependent variables which were LB11 concentrations and LB30 concentrations in the mixed solutions whilst in mono-dispersed system either LB11 concentrations or LB30 concentrations were used as dependent variables for composing the predictive models. The regression results of the predictive model are shown in figure 9.
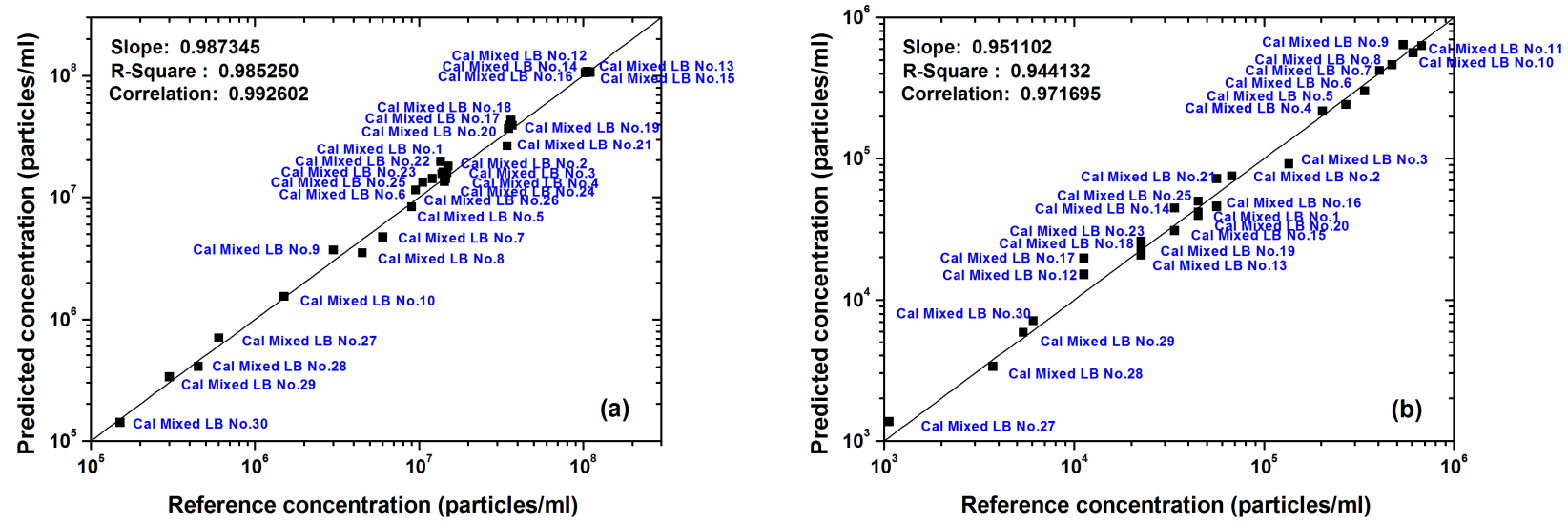

Figure 9 The results of the regression of the predictive models for (a) LB11 and (b) LB30 in polydispersion

The results of the regression of the predictive models in mixed suspensions as figure 9 show that the correlations between reference concentrations and predicted concentration of the calibration samples were 0.992 for LB11 and 0.972 for LB30 which meant the constructed models were able to be used as the predictive model. The angle dependent scattered intensities of the test samples were measured as figure 10a and analyzed for predicting concentration of LB11 and LB30 in the test samples using PLS-R method based on the constructed predictive model. The prediction results of test samples in mixed system are shown in figure $10 \mathrm{~b}$.
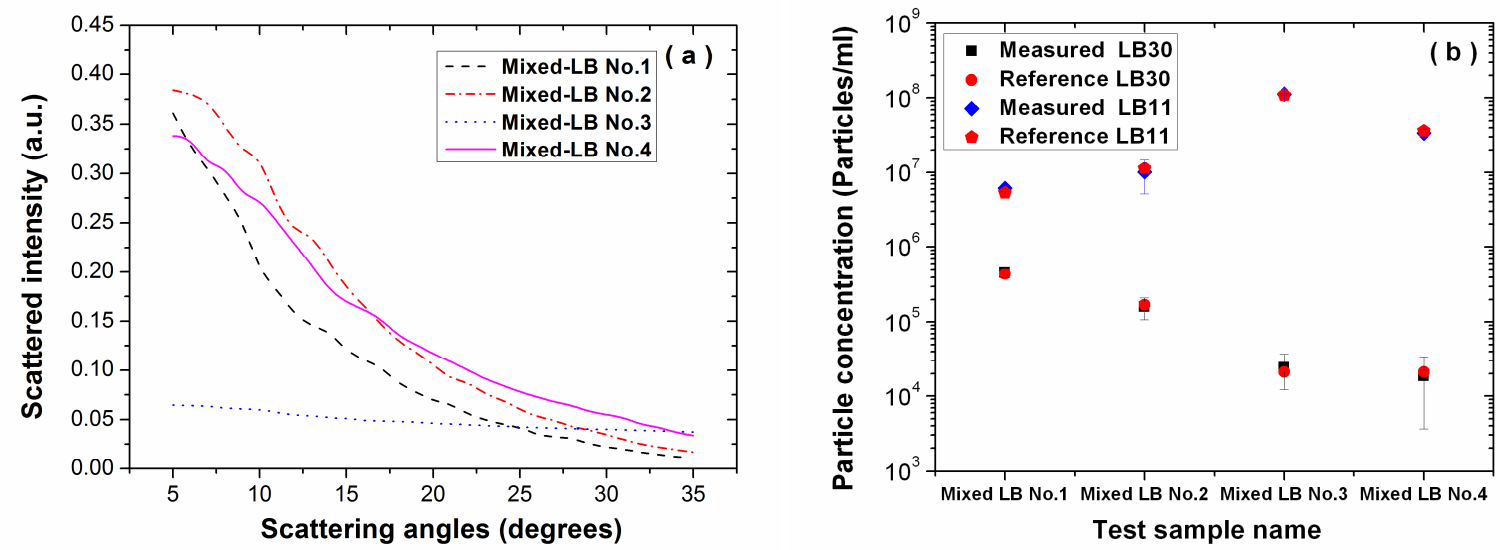

Figure 10 (a) Scattered intensities from test samples in poly-dispersed suspensions (b) Prediction results for test samples in poly-dispersed suspensions.

Figure $10 \mathrm{~b}$ shows predicted results for the poly-dispersed suspensions comprising $1.1 \mu \mathrm{m}$ and $3.0 \mu \mathrm{m}$ diameter particles. The predicted concentrations of each particle size were in agreement with the reference values. The predicted concentrations (particles/ml) and standard deviation in measurement (in bracket) of the test samples Mixed LB-No.1 to Mixed LB-No.4 were $6.08 \times 10^{6}\left(5.62 \times 10^{5}\right), \quad 1.01 \times 10^{7}\left(4.97 \times 10^{6}\right), \quad 1.12 \times 10^{8}\left(2.09 \times 10^{6}\right)$ and $3.37 \times 10^{7}$ $\left(4.25 \times 10^{6}\right)$ for the LB1 1 and $4.56 \times 10^{5}\left(5.96 \times 10^{4}\right), 1.59 \times 10^{5}\left(5.28 \times 10^{4}\right), 2.45 \times 10^{4}\left(1.21 \times 10^{4}\right)$ and $1.87 \times 10^{4}$ 
$\left(1.51 \times 10^{4}\right)$ for LB30 , respectively. The minimum error from the reference was $2.3 \%$ of concentration of $1.12 \times 10^{8}$ particles $/ \mathrm{ml}$ for LB11 and $3.5 \%$ of concentration of $4.56 \times 10^{5}$ particles $/ \mathrm{ml}$ for LB30. These results illustrated that the proposed system can be also used to measure the particle concentrations in poly-dispersed suspensions.

The results of both mono-dispersed and poly-dispersed system proved that the combination technique of the proposed scattering system with the partial least square regression method can be used to measure concentrations of particle suspensions in high concentration and in mixed suspensions. As this method uses a technique that uses patterns of the multiple-angle dependent scattered signals from the standard samples and statistical methods to predict the new observation concentrations, therefore this technique can be easily applied to systems which have a routine sample line.

\section{CONCLUSIONS}

A simple, multiple-angle light scattering system has been developed that can differentially measure the concentration of different sized particles in suspensions even in high concentration where the multiple scattering effects are significant. The system employs the multiple-angle dependent light scattering technique and partial least square regression method. It was validated by measuring concentrations of polystyrenes latex sphere aqueous suspension in mono and poly-dispersed suspension. The measurement results showed good agreement between the predicted results and the reference values. The approach that is described here represents a compact but effective system allowing multiple particle sizes even in high concentration to be measured simultaneously, such as for system to measure microorganism in water.

The authors acknowledge financial support from the Thai Royal Government.

\section{REFERENCES}

[1] Hidekazu Tai, Tsutomu Kobayashi and Kaoru Tsuzuki., "Measurement of Concentration in Particle Suspensions Using a Converged Ultrasonic Impulse with a Broad Frequency Bandwidth" Jpn. J. Appl. Phys. 44, 4443-4445 (2005).

[2] Tsutomu Kobayashi, Hidekazu Tai, Suguru Kato, "Measurement method of particle concentration and acoustic properties in suspension using a focused ultrasonic impulse radiated from a plano-concave transducer", Ultrasonics 44, 491-496 (2006).

[3] Gillian Carson, Anthony J. Mulholland, Alison Nordon, Manuel Tramontana, Anthony achagan, and Gordon Hayward. , "Estimating Particle Concentration Using Passive Ultrasonic Measurement of Impact Vibrations", IEEE Transactions on Ultrasonics Ferroelectrics and Frequency Control 56(2), (2009).

[4] M. Kostic, Vijay Kumar Sankaramadhi, and Kalyan Chaitanya Simham. , "New educational lab: Measurement and uncertainty evaluation of nanofluid particle concentration using volumetric flask method", American Society for Engineering Education, (2006).

[5] H.C. Van de Hulst., "Light Scattering by Small Particles", Wiley \& Sons, New York, (1957).

[6] H.C. Van de Hulst., "Multiple Light Scattering: Tables, Formulas, and Applications", Academic Press, New York., (1980).

[7] I Weiner, M Rust, T D Donnelly., "Particle size determination: An undergraduate lab in Mie scattering" American Journal of Physics, 69(2), (2001).

[8] Ben Veihelmann, Martin Konert, and Wim J. van der Zande., "Size distribution of mineral aerosol: using lightscattering models in laser particle sizing", Applied Optics, 45(23), 6022-6029 (2006).

[9] Jean-Luc Castagner and Irving J. Bigio., "Particle sizing with a fast polar nephelometer", Applied Optics, 46(4), 527532 (2007).

[10] Z. Ulanowski, R. S. Greenaway, P. H. Kaye, and I. K. Ludlow., "Laser diffractometer for single-particle scattering measurements", Meas. Sci. Technol. 13, 292-296 (2002).

[11] William D. Dicka; Paul J. Ziemannb; Peter H. McMurryc., "Multiangle Light-Scattering Measurements of Refractive Index of Submicron Atmospheric Particles" Aerosol Science and Technology 41, 549-569 (2007).

[12] Shaughnessy, E. J.; Morton, J. B., "Laser light-scattering measurements of particle concentration in a turbulent jet" Journal of Fluid Mechanics 80, 129-148 (1977). 
[13] Alfredo J. Armendariz , David Leith., "Concentration measurement and counting efficiency for the aerodynamic particle sizer 3320 " Aerosol Science 33, 133-148(2002).

[14] Simon Premože, Michael Ashikhmin, Ravi Ramamoorthi, Shree Nayar., "Practical Rendering of Multiple Scattering Effects in Participating Media" Eurographics Symposium on Rendering, (2004).

[15] Mokhtari T, Sorensen CM, Chakrabarti A., "Multiple-scattering effects on static light-scattering optical structure factor measurements." Appl Opt. 44(36), 7858-61 (2005).

[16] Dieter Lehner, Gerhard Kellner, Heimo Schnablegger and Otto Glatter., "Static Light Scattering on Dense Colloidal Systems: New Instrumentation and Experimental Results", J. Colloid and Interface Science 201(1), 34-47 (1998)

[17] Laurence Bergougnoux , Jean-Luc Firpo, Jacqueline Misguich-Ripault.; "Optical fiber sensor for measuring high particle concentrations", Powder Technology 105, 413-417(1999).

[18] F.H. Zhang, E. Lewis and P.J. Scully., "An optical fibre sensor for particle concentration measurement in water systems based on inter-fibre light coupling between polymer optical fibres", Transactions of the Institute of Measurement and Control 22(5), 413-430(2000).

[19] Yuko Yamaura, Takao Odake, Akifumi Suzuki, Mamiko Fujii and Kiyoshi Nakayama., "Experimental Study on Light Scattering of High Concentration Suspension", Opt. Rew 10(5), (2003).

[20] Anatoli P. Nefedov, Oleg F. Petrov, and Olga S. Vaulina., "Analysis of particle sizes, concentration, and refractive index in measurement of light transmittance in the forward-scattering-angle range", Applied Optics 36(6), (1997).

[21] Anshi Chen, Jiming Hao, Zhongping Zhou, and Kebin He., "Particulate concentration measured from scattered light fluctuations", Applied Optics 25 (10), (2000).

[22] Kleinbaum, David G., et al., "Applied Regression Analysis and Multivariable Method", Pacific Grove, Ca. Brooks/Cole Publishing Company, (1998). 